\title{
The Pregnant Breast Cancer Patient: Are We Failing Our Most Vulnerable Population?
}

\author{
Marie Catherine Lee, MD, FACS and Christine Laronga, MD, FACS \\ Department of Women's Oncology, H. Lee Moffitt Cancer Center, Tampa, FL
}

Breast cancer in the premenopausal population is uncommon, and breast cancer diagnosed in pregnancy is a frankly rare event, occurring in approximately $1 / 3,000$ breast cancer cases annually. Considering the recent national and international trend of deferred childbearing, the incidence of breast cancer in pregnancy is likely to remain the same or increase in the coming years. Happily, breast cancer patients have been the beneficiaries of cutting edge cancer therapy in all modalities. These advances have not only significantly improved quality of life, they have also had a marked effect on the longevity of women diagnosed with breast cancer, even those presenting with late-stage disease. Thus, it is our responsibility as surgeons and oncologists to offer this special population the best that modern medicine has on hand. However, we frequently fall short of this goal when treating the pregnant breast cancer patient due to a lack of knowledge, the paucity of data, as well as fear for the potential current and future effects of therapy on the fetus.

Although complicated by the timing of pregnancy, the data suggest that many of the surgical options recommended to non-pregnant patients should be considered for the gravid breast cancer patient, and termination of the pregnancy is by no means therapeutic. Given that the tumor biology seen in pregnant patients is similar to the nonpregnant premenopausal population, and that survival is dictated by tumor biology rather than the presence of a fetus, ${ }^{1,2}$ advocacy of the 'therapeutic abortion' is not only outdated but it borders on unethical. Unfortunately, the

(C) Society of Surgical Oncology 2014

First Received: 31 March 2014;

Published Online: 18 April 2014

M. C. Lee, MD, FACS

e-mail: marie.lee@moffitt.org myth of the 'therapeutic abortion' continues to persist in the medical community, even to this day.

For the newly-diagnosed pregnant patient, advances in breast imaging, particularly ultrasound, should not be forgotten. In particular, ultrasound of the axilla had demonstrated high sensitivity for axillary disease and, in combination with fine needle aspiration, a specificity of $100 \%$ for metastasis. ${ }^{3}$ The majority of patients diagnosed during their childbearing years present with palpable in-breast disease and many have concomitant gross axillary involvement; therefore, sonographic evaluation and needle aspiration of the axilla preoperatively should be strongly considered, particularly in the gravid population.

For the clinically node-negative pregnant patient, the practice of sentinel node biopsy is one that has been evaluated using non-pregnant human models in a variety of settings, primarily with Tc-99 lymphoscintigraphy, all of which have demonstrated clinically insignificant levels of potential radiation dose to the gravid uterus. ${ }^{4-8}$ One study has also examined the utility of methylene blue dye lymphoscintigraphy, although this has generally been avoided due to concerns of allergy and transplacental exchange. ${ }^{9}$ Although small datasets, these studies represent the only feasible prospective data due to the ethical and practical constraints of conducting research in a pregnant population.

In this issue of Annals of Surgical Oncology, Gropper et al. reviewed the 16 years single-institution experience of sentinel node biopsy in pregnancy. Their results mirror other small single-institution series supporting the safety and efficacy of sentinel node biopsy in pregnancy. ${ }^{6,10}$ However, this practice is not isolated; the National Comprehensive Cancer Network (NCCN) Breast Cancer guidelines suggest the discussion of sentinel node biopsy with Tc-99 in the setting of pregnancy and clinically nodenegative breast cancer. ${ }^{11}$ 
However, even more telling than the safety and utility of sentinel node biopsy in pregnancy is the actual utilization of lymphoscintigraphy in clinically node-negative breast cancer patients; in this cohort of 47 clinically node-negative pregnant patients, $42.6 \%$ of the group received upfront axillary dissection. The earliest patients in this cohort conceivably were treated in the early days of sentinel node biopsy, prior to the existing data regarding uterine nuclear dosing and the existing clinical series. However, the practice of upfront axillary node dissection in a clinically node-negative, non-pregnant population is most definitely outside the current accepted standard and, arguably, should not be considered a standard in the pregnant population given the growing body of literature.

Nevertheless, a recent American Society of Clinical Oncology practice guideline update regarding the use of sentinel node biopsy for early-stage breast cancer continues to advise against the use of sentinel node biopsy in the pregnant population. ${ }^{12}$ The authors note that the recommendation was made by informal consensus and is a weak recommendation. However, the publication of this as a practice guideline may further serve to confuse, rather than elucidate, the care of these uncommon and complex patients.

These contradictory viewpoints only serve to underscore the most important elements in the care of the pregnant breast cancer patient-informed discussions with the patient and a multidisciplinary care team. In most situations, the goal is not only optimal cancer care for a young breast cancer patient, but a healthy, full-term infant after an uncomplicated delivery. With these lofty, but achievable goals in mind, we strongly recommend that these women be evaluated in a multidisciplinary cancer setting with close integration of the patient's obstetrician and perinatologist. Open and informed communication among all parties is tantamount to the care of both patients involved in these rare but delicate situations.

\section{REFERENCES}

1. Middleton LP, et al. Breast carcinoma in pregnant women. Cancer. 2003;98(5):1055-1060.

2. Cardonick EMD, et al. Breast cancer during pregnancy: maternal and fetal outcomes. Cancer J. 2010;16(1):76-82.

3. Lee MC, et al. Consequences of axillary ultrasound in patients with T2 or greater invasive breast cancers. Ann Surg Oncol. 2011;18(1):72-77.

4. Gentilini $O$, et al. Sentinel lymph node biopsy in pregnant patients with breast cancer. Eur J Nucl Med Mol Imaging. 2010;37(1):78-83.

5. Gentilini O, et al. Safety of sentinel node biopsy in pregnant patients with breast cancer. Ann Oncol. 2004;15(9):1348-1351.

6. Keleher A, et al. The safety of lymphatic mapping in pregnant breast cancer patients using Tc-99m sulfur colloid. Breast $J$. 2004;10(6):492-495.

7. Spanheimer $P$, et al. Measurement of uterine radiation exposure from lymphoscintigraphy indicates safety of sentinel lymph node biopsy during pregnancy. Ann Surg Oncol. 2009;16(5):11431147.

8. Pandit-Taskar N, et al. Organ and fetal absorbed dose estimates from $99 \mathrm{mTc}$-sulfur colloid lymphoscintigraphy and sentinel node localization in breast cancer patients. J Nucl Med. 2006;47(7): 1202-1208.

9. Pruthi $\mathrm{S}$, et al. Pharmacokinetics of methylene blue dye for lymphatic mapping in breast cancer-implications for use in pregnancy. Am J Surg. 2011;201(1):70-75.

10. Khera SY, et al. Pregnancy-associated breast cancer patients can safely undergo lymphatic mapping. Breast J. 2008;14(3):250254.

11. Carlson R, et al. NCCN clinical practice guidelines in oncology: breast cancer. V.2.2010. NCCN; 2010.

12. Lyman GH, et al. Sentinel lymph node biopsy for patients with early-stage breast cancer: American Society of Clinical Oncology clinical practice guideline update. J Clin Oncol. Epub; 2014. 